\title{
A eficácia do Snoezelen na redução das estereotipias em adultos com deficiência intelectual: um estudo de caso da intervenção da terapia ocupacional em salas de estimulação multissensorial*
}

\author{
The effectiveness of Snoezelen in reducing stereotyping in \\ adults with intellectual disabilities: a case study of Occupational \\ Therapy intervention in multisensory stimulation rooms
}

\author{
Ana Sofia Pinto Lopes ${ }^{1}$, Janine Vanessa Martins Araújo ${ }^{1}$, \\ Marco Paulo Vieira Ferreira ${ }^{1}$, Jaime Emanuel Moreira Ribeiro ${ }^{2}$
}

http://dx.doi.org/10.11606/issn.2238-6149.v26i2p234-243

\begin{abstract}
Lopes ASP, Araújo JVM, Ferreira MPV, Ribeiro JEM. A eficácia do Snoezelen na redução das estereotipias em adultos com deficiência intelectual: um estudo de caso da intervenção da terapia ocupacional em salas de estimulação multissensorial. Rev Ter Ocup Univ São Paulo. 2015 maio-ago.;26(2):234-43.
\end{abstract}

RESUMO: Existem escassas evidências sobre a eficácia da intervenção em salas de Snoezelen na redução de estereotipias em adultos com deficiênciaintelectual. Nestesentido, opresente estudo pretendeu avaliar a relação entre esta estimulação multissensorial e a redução de estereotipias em adultos com deficiência intelectual. Por meio da metodologia de estudo de caso, analisou-se o comportamento de um sujeito antes, durante e após estimulação multissensorial em salas de Snoezelen, durante dez sessões bissemanais, com a duração de 1 h30. A recolha de dados foi concretizada por entrevista semiestruturada aos cuidadores formais do sujeito e da observação direta participante e não participante com registo audiovisual das sessões. Após a análise dos dados, com a utilização do software WebQDA, foi possível concluir que a estimulação multissensorial em sala de Snoezelen contribuiu para a redução das estereotipias no sujeito em estudo, durante a sua realização como imediatamente após, num contexto distinto. Outra evidência do presente estudo sugere que existe uma redução da frequência de estereotipias a médio prazo bem como o aumento da interação e comunicação do sujeito com o terapeuta.
DESCRITORES: Comportamento estereotipado; Deficiência intelectual; Terapia ocupacional.

Lopes ASP, Araújo JVM,Ferreira MPV, Ribeiro JEM. The effectiveness of Snoezelen in reducing stereotyping in adults with intellectual disabilities: a case study of Occupational Therapy intervention in multisensory stimulation rooms. Rev Ter Ocup Univ São Paulo. 2015 May-Aug.;26(2):234-43.

ABSTRACT: There is little evidence of the effectiveness of
intervention in Snoezelen rooms in stereotyping reduction
in adults with intellectual disabilities. In this direction, the
present study sought to evaluate the relationship between
this multisensory stimulation and stereotyping reduction
in adults with intellectual disabilities. Through the case
study methodology, the behavior of a subject was analyzed
before, during and after the multisensory stimulation in
Snoezelen rooms for ten biweekly sessions, lasting an hour
and a half. Data collection was completed by semi-structured
interviews with the formal caregivers of the subject and by
the participant and non-participant direct observation with
audiovisual recording sessions. After analyzing the data
using the WebQDA software, it was concluded that the
multisensory stimulation in Snoezelen rooms contributed to
reduce stereotyping in the subject being studied, both during
its realization and immediately after. Another evidence of
this study suggests that there is a reduction in the frequency

\footnotetext{
*Trabalho realizado na Escola Superior de Saúde de Leiria do Instituto Politécnico de Leiria no âmbito de projeto para conclusão da licenciatura em Terapia Ocupacional.

1. Alunos do $4^{\circ}$ ano do curso de licenciatura em Terapia Ocupacional da Escola Superior de Saúde de Leiria do Instituto Politécnico de Leiria.

2. Professor Adjunto Convidado do curso de licenciatura em Terapia Ocupacional; Coordenador do Departamento de Ciências e Tecnologias da Saúde da Escola Superior de Saúde - Instituto Politécnico de Leiria.

Endereço para correspondência: Doutor Jaime Emanuel Moreira Ribeiro. Instituto Politécnico de Leiria. Campus 2 - Morro do Lena Alto do Vieiro. Apartado 4137 | 2411-901 Leiria, Portugal. E-mail: jaime.ribeiro@ipleiria.pt
} 
of stereotyping in the medium term as well as increased interaction and communication between the subject and therapist.

\section{INTRODUÇÃO}

$\mathrm{A}$ deficiência intelectual é definida como um funcionamento intelectual global inferior à média da população. Este deve encontrar-se associado a déficits no comportamento adaptativo* e a alterações em pelo menos duas das seguintes áreas: comunicação, autocuidados, capacidades sociais e interrelacionais, uso de meios comunitários, capacidades acadêmicas, trabalho, lazer, saúde e segurança ${ }^{1}$.

Os indivíduos com deficiência intelectual apresentam, frequentemente, alterações no comportamento, como as estereotipias, manifestadas em cerca de $40 \%$ das pessoas em situação de institucionalização ${ }^{2}$. O comportamento estereotipado, ou estereotipias, é definido como um comportamento motor ou vocal repetitivo, rítmico e que não serve um propósito aparente ${ }^{3,4}$. Estas são usualmente associadas a atos motores ou vocais, podendo, ainda, estarem associadas à manipulação não funcional de objetos ${ }^{3,5}$.

As estereotipias motoras incluem movimentos corporais simples como balançar o corpo, agitar os braços, agitar os dedos, fazer caretas e o mover repetidamente várias partes do corpo, como as mãos, braços, pernas e olhos $^{3,6}$. As estereotipias vocais se caracterizam por sons vocais repetitivos, discurso repetitivo e obsessivo, ecolália, diálogo em scripting ${ }^{* *}$ e riso sem propósito social ${ }^{3,6}$.

Há consenso na literatura de que os comportamentos estereotipados interferem na aquisição de novas competências e inibem o desempenho ocupacional dos indivíduos ${ }^{3}$, aspecto fundamental na prática da Terapia Ocupacional. Estes são também apontados como fator que impede a interação social, que promove a estigmatização dos sujeitos e, em casos mais graves, pode ser percursor de autoagressão $0^{3,7}$.

Quando se verifica esse tipo de comportamentos, sua redução é assumida como prioridade da intervenção. Uma das abordagens de intervenção com indivíduos com deficiência intelectual é a terapia em Snoezelen, igualmente
KEYWORDS: Stereotyped behavior; Intellectual disability; Occupational Therapy.

utilizada em sujeitos com dificuldades de aprendizagem e distúrbios emocionais ${ }^{8,9}$.

O Snoezelen foi desenvolvido na Holanda, em 1975, e tem como objetivo promover a exploração do ambiente envolvente, o relaxamento mental e físico, os sentimentos de satisfação e as competências sociais ${ }^{8,9}$. Consiste no uso de equipamentos específicos, no interior de uma sala, que permite a exploração controlada dos diversos sentidos: tátil, visual, olfativo, auditivo, vestibular e propriocetivo ${ }^{2}$. Estes equipamentos podem promover a estimulação contingente, na qual o utilizador desenvolve um comportamento que irá desencadear uma resposta do equipamento, ou não contingente, em que este apenas recebe um estímulo e a sua resposta não apresenta qualquer alteração no ambiente ${ }^{11}$.

Entre os referidos equipamentos encontramse rádios, projetores, bolas de espelhos, fibras óticas, colchões de água, tubos de espuma, óleos de aromaterapia e balanços 9 .

As sessões realizadas nessas salas podem ser individuais ou de grupo, e o papel dos técnicos deve ser o de permitir e encorajar a interação dos participantes com o equipamento destinado à estimulação ${ }^{2,11}$.

Existem estudos que evidenciam a eficácia do Snoezelen na melhoria do comportamento. As evidências demonstram que os sujeitos se manifestam menos agressivos nas salas de estimulação multissensorial do que em outros ambientes. Um estudo elaborado por Shapiro et al. ${ }^{24} \mathrm{com}$ crianças com deficiência intelectual moderada e severa constatou a diminuição, em frequência e duração, dos comportamentos não adaptativos no Snoezelen, comparativamente às salas de brincar usuais ${ }^{11}$.

A investigação realizada por Matson et al. ${ }^{9}$ revelou também que o input sensorial recebido, quando da estimulação multissensorial em salas de Snoezelen, permite a redução das estereotipias, da autoagressão e de outros comportamentos considerados disruptivos. $\mathrm{O}$ estudo permite concluir que, imediatamente após a estimulação multissensorial, parece haver um aumento do envolvimento de indivíduos com deficiência intelectual

\footnotetext{
* Capacidade de adaptação às exigências de independência pessoal e responsabilidade social esperadas pelo grupo cultural no qual o indivíduo se encontra inserido ${ }^{10}$.

** Diálogo em scripting: repetição de diálogos a partir de programas televisivos ou filmes ${ }^{3,6}$.
} 
profunda e autismo na realização de tarefas propostas, bem como a diminuição da frequência global diária de comportamentos desafiadores. Outro estudo concluiu que os indivíduos preferem alguns estímulos em detrimento de outros e que a procura de estímulos preferenciais resulta numa alternativa que diminui comportamentos prejudiciais de autoestimulação ${ }^{12}$.

Cuvo et al. ${ }^{2}$ desenvolveram um estudo com crianças com deficiência intelectual profunda, e verificaram a redução de estereotipias e o aumento do envolvimento nas salas de Snoezelen comparativamente às salas de brincar. Outras investigações neste âmbito comprovam também que o uso de estímulos não contingentes pode reduzir efetivamente o comportamento estereotipado e o de autoagressão ${ }^{13}$.

Desta forma, a literatura científica existente evidencia a relação da terapia em sala de Snoezelen com a redução das estereotipias. No entanto são em número reduzido e apontam para a necessidade de se aprofundar a investigação nesta área, uma vez que as estereotipias são barreiras ao desempenho ocupacional e, portanto, à melhoria da qualidade de vida e da autonomia dos indivíduos. Neste sentido, a investigação realizada procura explorar e compreender a existência da relação direta entre a estimulação multissensorial em salas de Snoezelen e a redução dos comportamentos estereotipados em sujeitos com deficiência intelectual.

\section{PROCEDIMENTOS METODOLÓGICOS}

O presente estudo de caso do tipo qualitativo e exploratório/descritivo teve como objetivo avaliar a relação entre a estimulação multissensorial em salas de Snoezelen e a redução de estereotipias em adultos com deficiência intelectual. De forma mais específica, procurou avaliar a redução das estereotipias num indivíduo com deficiência intelectual durante e imediatamente após a estimulação multissensorial em sala de Snoezelen e se a redução se mantém num contexto distinto da sala.

A seleção do participante foi efetuada dentre sujeitos atendidos no Centro de Atividades Ocupacionais* da Organização de Apoio e Solidariedade para a Integração Social (OASIS), em Leiria, com diagnóstico de deficiência intelectual e presença de comportamentos estereotipados identificados como barreiras à sua participação ativa nas atividades significativas. Como critérios de exclusão destacam-se a presença de incapacidade motora associada, que impossibilite a exploração dos equipamentos de Snoezelen, e a ocorrência de episódios prévios de epilepsia ou surtos psicóticos ${ }^{* *}$.

O indivíduo selecionado tem 19 anos de idade, é do sexo masculino, com diagnóstico de Trissomia 21 do tipo Mosaico ${ }^{* * *}$. O sujeito reside com os pais e com dois irmãos e frequenta o Centro de Atividades Ocupacionais da OASIS. Nesta instituição, o participante realiza atividades estritamente ocupacionais, das quais cinco foram consideradas para a avaliação. Após consulta do processo individual do cliente verificou-se que o sujeito apresenta comportamentos estereotipados assinalados como inibidores ao seu desempenho ocupacional.

Para a concretização das sessões, foram usadas duas salas da instituição, uma de atividades e outra de Snoezelen (Figura 1). A sala de atividades selecionada encontra-se junto à sala de Snoezelen, possui em seu interior apenas uma escrivaninha e duas cadeiras, tem luz natural e artificial e conta com isolamento para ruídos externos. A sala de Snoezelen encontra-se equipada com um tapete de fibras ópticas, um colchão de água quente, um colchão com duas colunas de água, um balanço, espelhos, projetores e sistema de som.

O estudo foi desenvolvido por meio de dez sessões bissemanais, com a duração total de $1 \mathrm{~h} 30 \mathrm{~m}$, perfazendo um total de cinco semanas, decorridas nos meses de abril e maio de 2014. A primeira sessão foi usada para avaliar o comportamento do participante nas salas de atividade e de Snoezelen e para realizar um levantamento das atividades estritamente ocupacionais ${ }^{* * * *}$ desempenhadas diariamente pelo mesmo. As demais sessões foram divididas em três fases: nos primeiros 30 minutos realizou-se uma atividade numa sala de atividades ( $1^{\mathrm{a}}$ fase), nos 30 minutos seguintes realizou-se uma sessão de estimulação multissensorial em sala de Snoezelen ( $2^{\mathrm{a}}$ fase $)$ e, por fim, nos restantes 30

\footnotetext{
* Resposta social que tem como principal objetivo promover e disponibilizar condições que contribuam para a qualidade de vida da pessoa com deficiência e incapacidade, por meio do desempenho de atividades ocupacionais ${ }^{14}$.

** De acordo com a literatura científica, os estímulos visuais e luminosos podem provocar novas crises de epilepsia, e não é recomendado o excesso de estimulação em indivíduos com antecedentes de atividade delirante e alucinatória ${ }^{15}$.

*** A síndrome de Down do tipo mosaico constitui 1\% dos casos de síndrome de Down e resulta de uma alteração na disjunção mitótica do cromossomo 21. Estes sujeitos possuem uma mistura de células com 46 cromossomos e outras com 47 e as suas manifestações clínicas dependem da quantidade de células com o número de cromossomos anormais ${ }^{16}$.

**** Atividades que visam manter a pessoa ativa e interessada, favorecendo o seu equilíbrio e bem-estar físico, emocional e social ${ }^{14}$.
} 
minutos efetuou-se novamente a atividade inicial ( $3^{\mathrm{a}}$ fase). Uma vez que o contexto influencia o comportamento dos indivíduos, as terceiras sessões das atividades foram realizadas em dois contextos distintos. Assim, nas primeiras cinco sessões o participante permaneceu na sala de Snoezelen, (com os estímulos desligados), enquanto que nas restantes esse foi encaminhado novamente para a sala de atividades.

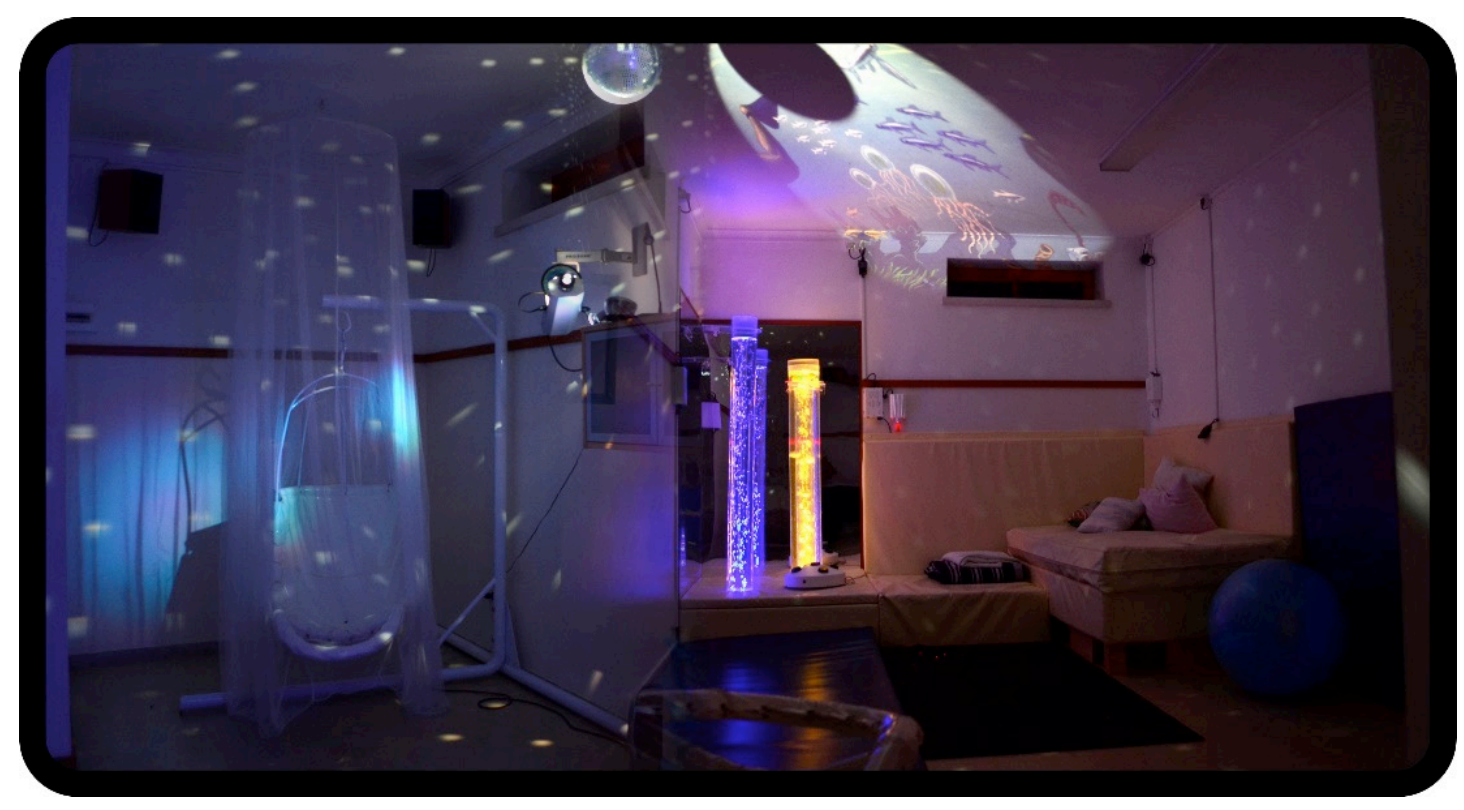

Figura 1 - Sala de Snoezelen da instituição OASIS

Relativamente aos métodos de coleta de dados, procedeu-se a uma entrevista semiestruturada com os cuidadores formais do participante, para conhecimento prévio das atividades a serem desenvolvidas no estudo, à observação indireta, por meio da consulta do processo individual do cliente, e a observação direta participante e não participante das gravações audiovisuais de todas as sessões. Para possibilitar a coleta de dados foi pedido o consentimento livre e informado aos tutores legais do sujeito e a autorização à instituição OASIS.

O processamento dos dados foi realizado por meio de análise quantitativa de conteúdo. Este consiste num método estruturado, baseado em princípios da ciência social, de medição e contagem que pode ser usado na análise de conteúdo, nomeadamente entrevistas, áudio e vídeo. O objetivo desse método de análise é examinar uma grande quantidade de informação qualitativa por métodos estatísticos ${ }^{17}$. Para o referido efeito, foi utilizado o software WebQDA, que permitiu a análise de dados audiovisuais ${ }^{18}$. De modo a reduzir possíveis enviesamentos e visando à validação destes dados, recorreu-se à triangulação de investigadores. Nesta, três dos investigadores analisaram individualmente as filmagens de todas as sessões e registraram a quantidade de estereotipias motoras observadas. Para o estudo, foi utilizada a média dos valores alcançados.

\section{RESULTADOS/DISCUSSÃO}

Após realizada a primeira sessão, constatou-se que o indivíduo possui várias estereotipias que se incluem na categoria das motoras. Das observadas, destacam-se tocar repetidamente: com o primeiro dedo na região do mento; com o segundo dedo na zona temporal; com o segundo dedo na zona zigomática; com o primeiro dedo nos membros inferiores; com o terceiro dedo nas sobrancelhas; com a polpa dos dedos na região torácica e com o bordo cubital de uma das mãos no membro superior oposto. Para além destas, incluem-se o fazer caretas sem propósito, abrir e fechar a boca repetidamente e balançar com a cabeça juntando ambas as mãos à face. Ainda nessa sessão, foi possível concluir que o sujeito executa simultaneamente mais do que uma das estereotipias citadas. Relativamente ao comportamento do sujeito na sala de Snoezelen, verificou-se que esse aparentou desconforto no colchão de água e no balanço. 
Tal comportamento pode estar associado aos déficits na integração sensorial", nomeadamente nos sistemas vestibular $^{* *}$ e táti $^{* * *}$, fatores também anteriormente assinalados em seu processo clínico. A disfunção na integração de estímulos vestibulares provoca insegurança gravitacional, que se traduz no medo excessivo ou desproporcionado de estímulos vestibulares, enquanto que alterações na integração de estímulos táteis resultam em defesa tátil manifestada em respostas emocionais excessivas ou aversivas quando o indivíduo é tocado inesperadamente ou quando é sujeito a qualquer contato físico ${ }^{19}$. Assim, uma vez que o balanço e o colchão de água provocam um aporte de estímulos ao sistema vestibular e tátil, o sujeito revelou dificuldades em sua utilização, pedindo, algumas vezes, a interrupção. No entanto, ao longo das sessões foi possível verificar um aumento da aceitação ao colchão de água.

Ainda na sessão de avaliação, bem como no decorrer do estudo, o sujeito manifestou preferência por estímulos visuais, nomeadamente os emitidos nas colunas de água (bolhas e luzes com diferentes cores intercaladas) e verificou-se que a presença ou ausência dos estímulos auditivos não provocaram qualquer alteração em seu comportamento. Tais resultados vão ao encontro do estudo elaborado por Matson et al. ${ }^{9}$, que confirma existir uma preferência pelos estímulos visuais em geral, contrariamente aos estímulos auditivos que parecem não ter qualquer efeito no comportamento dos indivíduos.

Relativamente às sessões que integram o estudo, é possível observar no Gráfico 1 que a sessão na qual se verificou maior frequência de comportamentos estereotipados foi a sessão 1, com uma percentagem de $62,83 \%$ na primeira fase e $59,13 \%$ na terceira, enquanto que na sessão 7 se observou a menor percentagem dos comportamentos supracitados, com $41,02 \%$ e $19,83 \%$, nas primeira e terceira fases, respectivamente. O Gráfico 2 corrobora estes resultados, na medida em que a média da sessão 1 apresentou a maior percentagem de estereotipias, com um valor de $56,66 \%$, e a sessão 7 apresentou o valor menor com 37, 42\%. Na sessão 6 ocorreu a maior redução da percentagem de estereotipias da primeira para a terceira fase, de $48,47 \%$ para $18,83 \%$.
Ainda de acordo com o Gráfico 2, nas sessões 1,6 7 9 e 5 verificaram-se percentagens superiores de frequência de estereotipias nas primeiras fases, quando comparadas às terceiras. Por outro lado, nas sessões $2,3,4,8$ e 10, a percentagem de comportamentos estereotipados foi superior nas terceiras fases comparativamente com as primeiras.

Ainda na análise do Gráfico 1, os comportamentos estereotipados do sujeito apresentaram redução mais acentuada nas sessões em que a terceira fase foi realizada na sala de atividades (sessões 6, 7, 8 e 9), analogamente com a $3^{\text {a }}$ fase das sessões que decorreram na sala de Snoezelen (sessões 1,2,3 e 4). O mesmo pode ser observado no Gráfico 3 em que a percentagem da média dos comportamentos estereotipados, destas sessões, reduziu de $52,16 \%$ para $43,81 \%$.

Ainda de acordo com o Gráfico 3 , nas cinco primeiras sessões observa-se o aumento dos comportamentos estereotipados da primeira fase para a terceira, cuja percentagem da média aumentou de $49,19 \%$ para $52,16 \%$. Nas restantes sessões verificou-se o oposto, com uma diminuição da percentagem da média de comportamentos com estereotipias de $46,7 \%$ para $43,81 \%$.

Esses dados indicam que parece existir uma relação do contexto onde se realiza a atividade com a presença de comportamentos estereotipados, uma vez que o comportamento do sujeito foi variável de acordo com o local onde foram realizadas as atividades da terceira fase da investigação. Quando as atividades se realizaram na sala de Snoezelen, com os estímulos desligados, contabilizaramse mais comportamentos estereotipados do que quando foram desempenhadas no contexto inicial do estudo. Este fato poderá ser explicado pela interferência que a presença dos equipamentos que fornecem os estímulos provoca na atenção e concentração do sujeito para a realização das atividades.

Pela observação do Gráfico 1 , as $1^{\mathrm{a}} \mathrm{s}$ fase das sessões 6,7,8 e 10 apresentam menor percentagem de estereotipias comparativamente à $1^{\mathrm{a}}$ fase das sessões $1,2,3$ e 5 , excetuando o verificado na atividade 4 , na qual ocorreu um aumento de estereotipias da $1^{\mathrm{a}}$ fase da sessão 4 para a $1^{\text {a }}$ fase da sessão 9 .

\footnotetext{
* Processo pelo qual o Sistema Nervoso Central organiza os estímulos sensoriais com o objetivo de fornecer respostas adaptativas às exigências do ambiente ${ }^{20}$.

** Este sistema envia informações ao sistema nervoso central sobre a posição e movimento da cabeça, a direção da gravidade, estabiliza o olhar durante os movimentos, controla os ajustes posturais ${ }^{20}$.

*** Fornece informação sobre estímulos externos que entram em contacto com a pele, a temperatura, a textura, o formato e o deslocamento dos objetos sobre o corpo. Este sistema juntamente com os sistemas proprioceptivo e vestibular fornecem as sensações básicas para o desenvolvimento da consciência corporal, fundamental para uma adequação das respostas ao meio ${ }^{21}$.
} 
Gráfico 1 - Estereotipias apresentadas durante as atividades

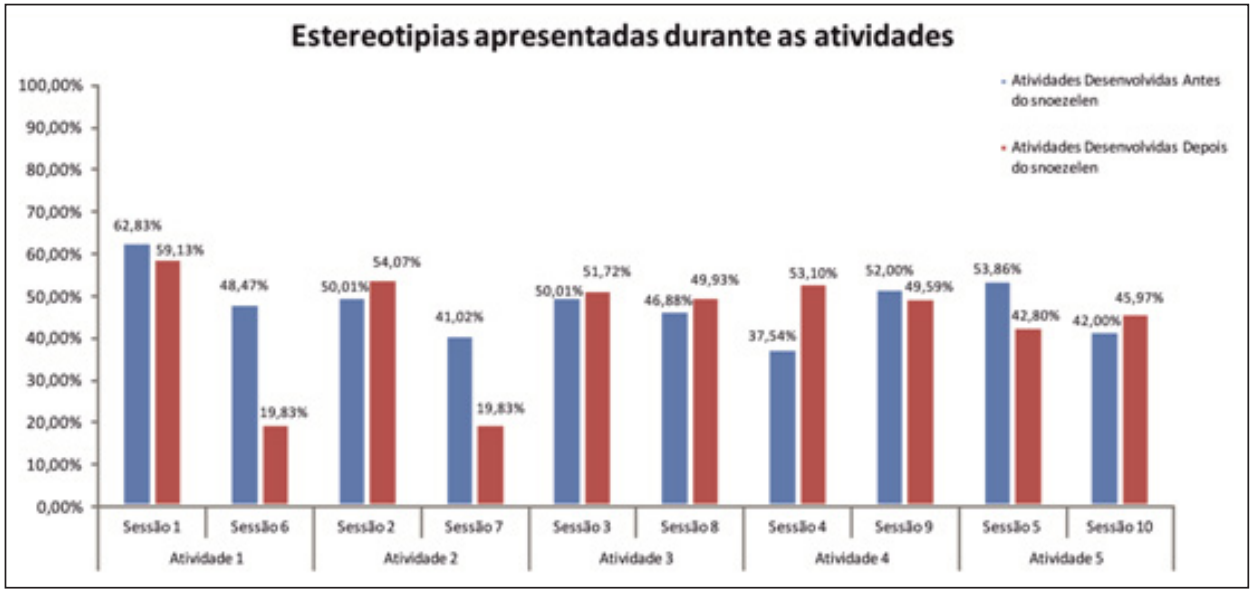

Gráfico 2 - Médias de comportamentos observados

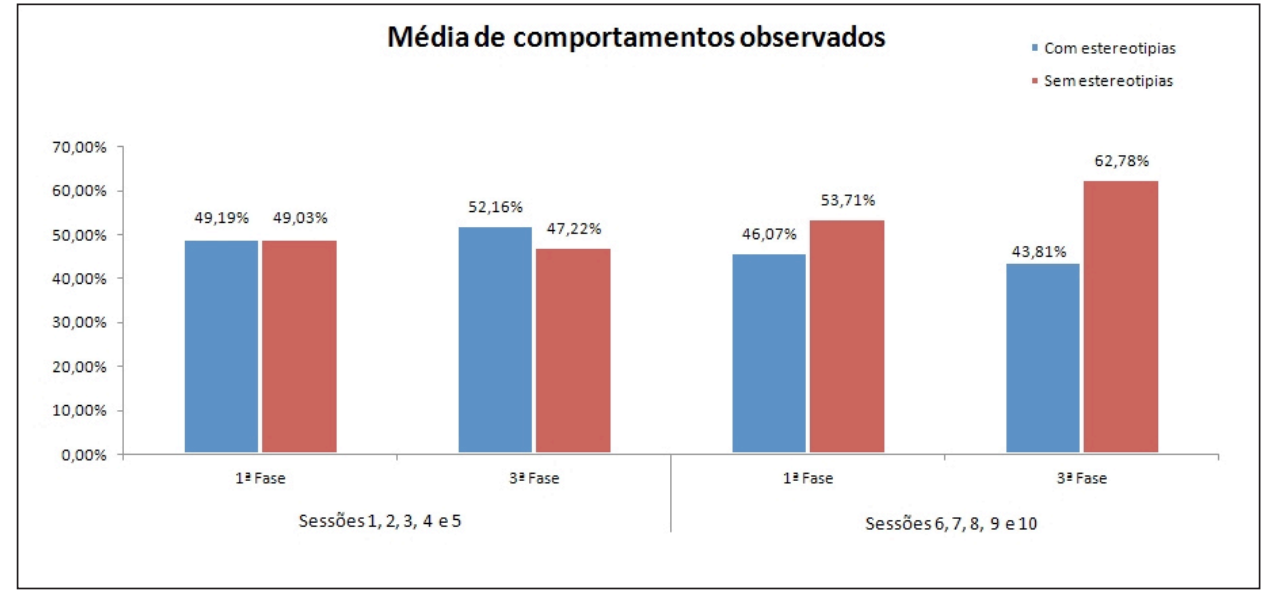

Gráfico 3 - Médias por sessão

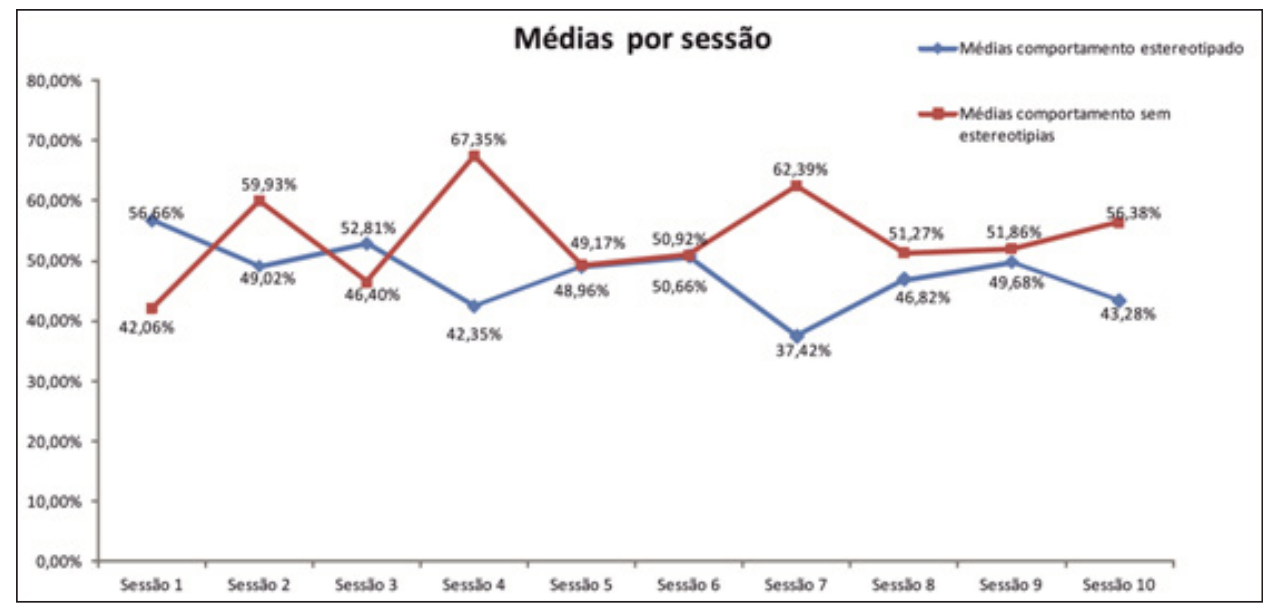


Comparando a primeira fase das cinco sessões iniciais com a correspondente das últimas cinco, de acordo com o Gráfico 3, verifica-se uma diminuição da percentagem dos comportamentos estereotipados, de $49,19 \%$ para $46,07 \%$. Relativamente à terceira fase das sessões, manifesta-se notoriamente uma diminuição de $8,35 \%$ da percentagem de comportamentos estereotipados das sessões $1,2,3,4$ e 5 para as sessões 6,7,8,9 e 10 .

Pela análise do Gráfico 2, da primeira para a última sessão do estudo manifesta-se uma diminuição de 13,38\% do comportamento estereotipado. No mesmo gráfico é possível verificar que, a partir da sessão 4 , o comportamento sem estereotipias mantém-se regularmente superior ao comportamento estereotipado.

Tais evidências sugerem que a estimulação multissensorial em salas de Snoezelen realizada frequentemente pode ter efeitos a médio prazo na redução de estereotipias em adultos com deficiência intelectual. A análise do comportamento do sujeito na sala de Snoezelen também suporta esta ideia, uma vez que, pela observação do Gráfico 4, se pode constatar que a percentagem de comportamentos estereotipados diminui em $6,14 \%$ da primeira sessão para a última.

Gráfico 4 - Intervenção no Snoezelen com estímulos ligados

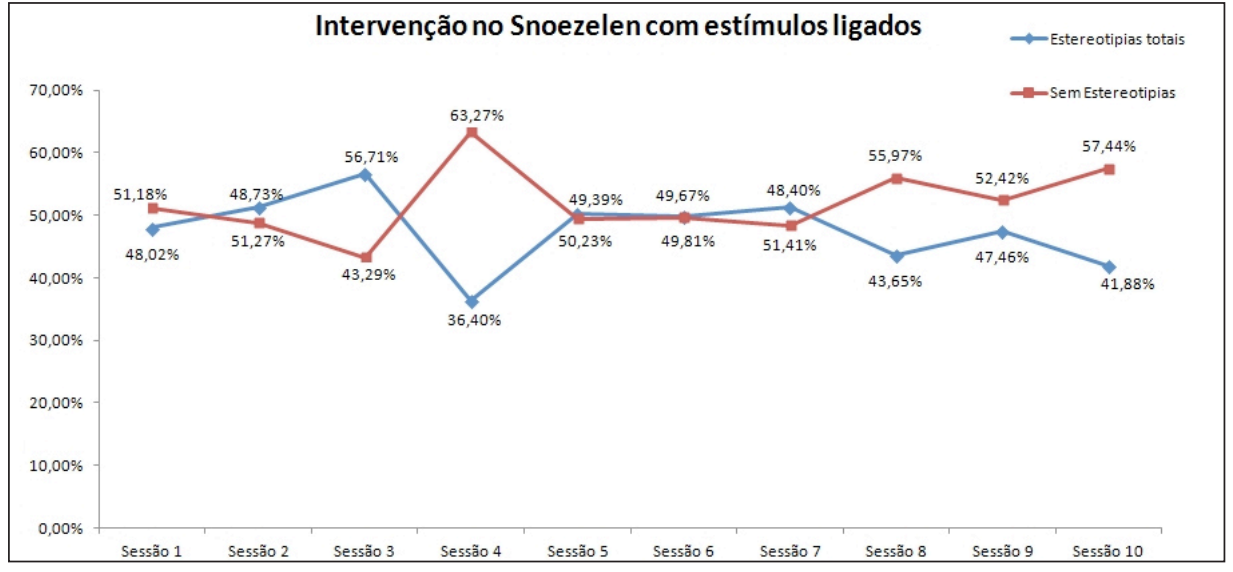

Apesar disto, pela análise do Gráfico 1 pode ainda verificar-se que a partir da sessão 8 , inclusivamente, os comportamentos estereotipados voltam a sofrer um aumento em todas as fases das sessões. Este fato pode ser explicado pela alteração do protocolo de intervenção, uma vez que, por falta de assiduidade do sujeito, as referidas sessões tiveram que ser realizadas na mesma semana. Este acontecimento, devido aos constrangimentos mencionados, pode ter suscitado uma regressão dos comportamentos do indivíduo, justificando os resultados obtidos.

Relativamente às estereotipias identificadas, após o estudo concluiu-se que a estereotipia mais frequente nas atividades foi o tocar repetidamente com o primeiro dedo na zona do mento, seguida do balançar a cabeça juntando ambas as mãos à face e da combinação de estereotipias. Nestas, as menos frequentes foram o tocar repetidamente com o primeiro dedo nos membros inferiores, tocar repetidamente com a polpa dos dedos na região toráxica e tocar repetidamente com o segundo dedo na zona zigomática.
A estereotipia mais frequente na sala de Snoezelen foi o balançar a cabeça com ambas as mãos junto à face, tocar repetidamente com o primeiro dedo nos membros inferiores, tocar repetidamente com o primeiro dedo na região do mento, passar o terceiro dedo nas sobrancelhas, seguidas da combinação de estereotipias. As menos frequentes foram tocar repetidamente com o segundo dedo na zona zigomática, fazer caretas e tocar repetidamente com a polpa dos dedos na região torácica.

A estereotipia tocar repetidamente com o primeiro dedo na região do mento apresentou uma diminuição gradual ao longo das sessões, tendo sido especialmente observável nas terceiras fases das sessões, quando de sua realização na sala de atividades.

No que diz respeito ao tocar repetidamente com o dedo na zona zigomática, esta não se verificou nas terceiras fases de todas as sessões.

A estereotipia tocar repetidamente com a polpa dos dedos na região torácica apenas se verificou na primeira fase das primeiras cinco sessões. 
Balançar a cabeça com ambas as mãos junto à face foi diminuindo ao longo das sessões, verificando-se menor frequência quando as terceiras fases foram realizadas na sala de atividades. Na sala de Snoezelen, esta verificouse principalmente durante a interação do sujeito com o terapeuta no tapete com fibras ópticas. Ao longo do estudo, foi possível concluir que esta estereotipia ocorreu maioritariamente após reforço positivo fornecido pelo terapeuta aquando do desempenho satisfatório das tarefas.

Quanto ao tocar com os dedos nos membros inferiores, deixou de se observar durante a realização das atividades nas sessões 6,7,8,9 e 10. Na sala de Snoezelen, esta foi mais frequente no tapete e nas colunas de água e não ocorreu no balanço, tendo sido verificada uma redução gradual até à inexistência de registros na sessão 10 .

A estereotipia abrir e fechar a boca sofreu um aumento nas terceiras fases das sessões 1,2,3,4 e 5, tendo diminuído nas fases respetivas das sessões 6,7,8,9 e 10 .

Relativamente à estereotipia passar com o terceiro dedo nas sobrancelhas teve uma maior diminuição nas terceiras fases das sessões $1,2,3,4$ e 5 , no entanto verificou-se um aumento nas sessões 6,7,8,9 e 10. Na sala de Snoezelen esta verificou- se em todos os estímulos, à exceção do balanço, e foi mais frequente quando o terapeuta deixava de interagir com o sujeito.

A combinação de estereotipias é desencadeada quando o terapeuta deixa de interagir com o sujeito e sofre um aumento nas terceiras fases de todas as sessões.

Por fim, a estereotipia tocar repetidamente com o bordo cubital de uma mão no membro superior oposto sofreu uma diminuição gradual ao longo das dez sessões.

Ao longo do estudo, foi ainda possível constatar que durante a realização das atividades o sujeito apresentou comportamentos estereotipados entre o desempenho de tarefas distintas. No entanto, ocorreu uma diminuição da frequência desses comportamentos com o decorrer do estudo, já que o indivíduo passou a realizar mais do que uma tarefa sem que isto se verificasse.

Outro facto relevante observado ao longo do estudo foi o aumento da capacidade de resposta perante solicitações de interação e comunicação do terapeuta, bem como a iniciativa do mesmo para a comunicação verbal e não verbal. Para além disso, manifestou-se um aumento da receptividade do sujeito ao contato físico com o terapeuta, tendo existido momentos em que foi o próprio a iniciar os referidos contatos. O mesmo foi observado em outro estudo realizado por Hutchinson e Haggar (1991), suportando a ideia de que a estimulação multissensorial em salas de Snoezelen incentiva a interação e as relações satisfatórias entre o cliente e o facilitador ${ }^{22}$.

Verificou-se que o balanço e o colchão de água, com as luzes ligadas, foram os equipamentos nos quais menos estereotipias ocorreram, contrariamente ao tapete, equipamento que desencadeou mais comportamentos estereotipados. Esses resultados convergem para a teoria da existência de estereotipias como autoestimulação sensorial*, uma vez que tais comportamentos verificaram-se em menor frequência nos equipamentos que fornecem menor input sensorial ${ }^{23}$.

\section{CONCLUSÕES}

O estudo aqui descrito encetou um caminho ainda por desbravar, limitado por condicionantes sempre associadas à população em estudo, nomeadamente a consciencialização da responsabilidade pela colaboração com a investigação científica por parte dos tutores do participante. Constrangimentos de assiduidade do participante acarretaram alterações ao protocolo de investigação inicialmente previsto, que, no entanto, não limitaram o estudo, mas o ampliaram. Como apanágio da investigação que integra a componente qualitativa, obtiveram-se dados não inicialmente perscrutados, mas que abrem novas perspetivas sobre os resultados da investigação de cariz multissensorial.

Estudos futuros de natureza semelhante podem explorar a redução do tempo das sessões, já que estas podem ter sido, porventura, demasiado longas, o que pode ter levado à exaustão do sujeito. Apesar dessas ilações, foi possível concluir que a estimulação multissensorial em sala de Snoezelen contribuiu efetivamente para a redução das estereotipias no sujeito em estudo, tanto durante sua realização como imediatamente após, num contexto distinto da sala citada.

Observou-se, também, a médio prazo, uma redução dos comportamentos estereotipados e um aumento da interação e da comunicação verbal e não verbal do sujeito com o terapeuta. Estes fatos culminam na hipótese de se tratar de uma abordagem de intervenção efetiva da Terapia Ocupacional para a população estudada, ressalvando que, tendo em conta a reduzida amostra do presente estudo, não se podem generalizar os resultados obtidos. Para além

\footnotetext{
* A função das estereotipias é a de fornecer estimulação sensorial adicional para substituir sensações ausentes ou incrementar modalidades sensoriais preservadas ${ }^{23}$.
} 
Lopes ASP, et al. A eficácia do Snoezelen na redução das estereotipias. Rev Ter Ocup Univ São Paulo. 2015 maio/ago.;26(2):234-43.

disso, é importante ressalvar que outros equipamentos de Snoezelen não utilizados nesta investigação podem suscitar diferentes respostas no indivíduo em estudo. Assim, e apesar de este ter sido um estudo inovador pelos resultados a médio prazo que obteve, trata-se de um projeto inacabado, com uma diversidade de opções por explorar, o que leva-nos a afirmar a necessidade da realização de mais pesquisas neste âmbito, que para efeitos de generalização beneficiariam de amostras mais significativas.

\section{REFERÊNCIAS}

1. American Psychiatric Association. Diagnostic and statistical manual of mental disorders: DSM-IV-T.R. 4th ed. Washington DC; 2000.

2. Cuvo A, May M, Post T. Effects of living room, Snoezelen room, and outdoor activities on stereotypic behavior and engagement by adults with profound mental retardation. Res Dev Disabil. 2001;22(3):183-204. DOI:10.1016/ S0891-4222(01)00067-1

3. Gardenier N, MacDonald R, Green G. Comparison of direct observational methods for measuring stereotypic behavior in children with autism spetrum disorders. Res Dev Disabil. 2004;25(2):99-118. DOI:10.1016/j. ridd.2003.05.004.

4. Ruggieri V, Caraballo R, Arroyo H. Temas de neuropediatria. Buenos Aires: Panamericana; 2005.

5. Falcomata TS, Roane HS, Feeney BJ, Stephenson KM. Assessment and treatment of elopement maintained by access to stereotypy. J Appl Behav Anal. 2010;43(3):513-7. DOI:10.1901/jaba.2010.43-513

6. Tarbox J, Kenzer A, Bishop M, Kakavand H, Wilke A, Dixon D. Indirect functional assessment of stereotypy in children with autism spectrum disorders. Res Autism Spectr Disord. 2011;6(2):824-8. DOI:10.1016/j. rasd.2011.11.003.

7. Reed F, Hirst J, Hyman S. Assessment and treatment of stereotypic behavior in children with autism and other developmental disabilities: a thirty year review. Res Autism Spectr Disord. 2011;6(1):422-30. DOI:10.1016/j. rasd.2011.07.003.

8. Lotan M, Gold C. Meta-analysis of the effectiveness of individual intervention in the controlled multisensory environment (Snoezelen) for individuals with intellectual disability. J Intellect Dev Disabil. 2009;34(3):207-15. DOI:10.1080/13668250903080106.

9. Matson J, Bamburg J, Smalls Y. An analysis of Snoezelen equipment to reinforce persons with severe or profound mental retardation. Res Dev Disabil. 2004;25(1):89-95. DOI:10.1016/j.ridd.2003.10.001
10. Youhua W, Thomas O, James A, MacLean W. Multigroup Confirmatory Factor Analysis for the Adaptive Behavior Assessment System-II Parent Form, Ages 5-21. Am J Ment Retard. 2008;113(3):178-86. DOI: 10.1352/0895-8017.

11. Hill L, Trusler K, Furniss F, Lancioni G. Effects of multisensory environments of stereotyped behaviours assessed as maintained by automatic reinforcement. J Appl Res Intellect Disabil. 2012;25(6):509-21. DOI:10.1111/ J.1468-3148.2012.00697.x

12. Rozen A. The effects of a snoezelen environment on a seven-year-old male with severe autism [Tese]. Toronto: York University; [s.d] [cited 2014 Feb 12]. Available from: //individual.utoronto.ca/abbyrozen/thesis.html.

13. Martin N, Gaffan E, Williams T. Behavioural effects of long-term multi-sensory stimulation. Br J Clin Psychol. 1998;37(1):69-82. DOI:10.1111/j.2044-8260.1998.tb01280.x

14. Instituto Segurança Social. Manual de processos-chave: centro de atividades ocupacionais. Lisboa. [acesso em 18 jan. 2014]. Disponível em: http://www4.seg-social.pt/ documents/10152/13337/gqrs_cao_processos-chave.

15. Hope KW. The effects of multisensory environments on older people with dementia. J Psychiatr Ment Health Nurs. 1998;5(5):377-85. DOI:10.1046/j.1365-2850.1998.00143.x

16. Kumar V, Abbas A, Aster J. Robbins: patologia básica. Rio de Janeiro: Elsevier; 2013.

17. Neuendorf K. A. The content analysis guidebook. Cleveland: Sage; 2002.

18. Souza F, Costa A, Moreira A, Souza D. WebQDA software de apoio à análise qualitativa: manual do utilizador. Aveiro: Universidade de Aveiro; 2013.

19. Kleiner A, Schlittler D, Sanchez-Arias M. O papel dos sistemas visual, vestibular, somatosensorial e auditivo para o controle postural. Rev Neurocienc. 2011;19(2): 349-57. Disponível em: http://www.revistaneurociencias.com. br/edicoes/2011/RN1902/revisao\%2019\%2002/496\%20 revisao.pdf.

20. Antunes E, Vicentini C. Desenvolvendo a sensibilidade sensorial tátil plantar em portadores de autismo infantil 
Lopes ASP, et al. A eficácia do Snoezelen na redução das estereotipias. Rev Ter Ocup Univ São Paulo. 2015 maio/ago.;26(2):234-43.

através do "tapete sensorial" - estudo de três casos. Cad Ter Ocup UFSCar. 2005;13(1):47-52. Disponível em: http:// www.cadernosdeterapiaocupacional.ufscar.br/index.php/ cadernos/article/view/177/134.

21. Neistadt M, Crepeau E. Willard \& Spackman terapia ccupacional. 9th ed. Rio de Janeiro: Guanabara Koogan; 2002.

22. Lotan M, Shapiro M. Management of young children with Rett disorder in the controlled multi-sensory (Snoezelen) environment. Brain Dev. 2005;27:88-94. DOI:10.1016/j. braindev.2005.03.021

23. Tuchman R, Rapin I. Autismo: abordagem neurobiológica. Porto: Artmed; 2009.

24. Shapiro M, Parush S, Green M, Roth D. The efficacy os the "Snoezelen" in the management of children with mental retardation who exhibit maladaptative behaviours. Br J Develop Desabil. 1997;43(85 pt 2):140-55. Available from: http://contents.bjdd.net/oldPDFs/85_140to155.pdf.

Recebido para publicação: 08.02.15

Aceito para publicação: 31.05 .15 